\title{
Chronic systemic pesticide exposure reproduces features of Parkinson's disease
} \author{
and J. Timothy Greenamyre \\ Department of Neurology, Emory University, 1639 Pierce Drive, WMB 6000, Atlanta, Georgia 30322, USA \\ The first two authors contributed equally to this work \\ Correspondence should be addressed to J.T.G. (jgreena@emory.edu)
}

Ranjita Betarbet, Todd B. Sherer, Gillian MacKenzie, Monica Garcia-Osuna, Alexander V. Panov

\begin{abstract}
The cause of Parkinson's disease (PD) is unknown, but epidemiological studies suggest an association with pesticides and other environmental toxins, and biochemical studies implicate a systemic defect in mitochondrial complex I. We report that chronic, systemic inhibition of complex I by the lipophilic pesticide, rotenone, causes highly selective nigrostriatal dopaminergic degeneration that is associated behaviorally with hypokinesia and rigidity. Nigral neurons in rotenone-treated rats accumulate fibrillar cytoplasmic inclusions that contain ubiquitin and $\alpha$-synuclein. These results indicate that chronic exposure to a common pesticide can reproduce the anatomical, neurochemical, behavioral and neuropathological features of PD.
\end{abstract}

Parkinson's disease is a late-onset, progressive motor disease marked by selective degeneration of dopaminergic neurons of the substantia nigra and formation of fibrillar cytoplasmic inclusions, known as Lewy bodies, which contain ubiquitin and $\alpha$-synuclein ${ }^{1}$. Rare cases of familial PD have been linked to mutations in $\alpha$-synuclein or parkin ${ }^{2-4}$, but the cause of the more commonly encountered sporadic disease is unknown, and the role of genetics in these cases is uncertain ${ }^{5}$. Post mortem studies strongly implicate oxidative damage and mitochondrial impairment in the pathogenesis of $\mathrm{PD}^{6}$. Epidemiological studies have suggested that pesticide exposure is associated with an increased risk of developing $\mathrm{PD}^{7-9}$.

After the pro-toxin N-methyl-4-phenyl-1,2,3,6-tetrahydropyridine (MPTP) was reported to produce in humans an acute parkinsonian syndrome that is virtually indistinguishable from idiopathic $\mathrm{PD}^{10}$, its metabolite, 1-methyl-4-pyridinium $\left(\mathrm{MPP}^{+}\right)$, was found to be a mitochondrial poison that inhibits mitochondrial respiration at complex I of the electron transport chain $^{11,12}$. The selectivity of $\mathrm{MPP}^{+}$for dopaminergic neurons is due to the fact that it is an excellent substrate for the dopamine transporter, and is thereby accumulated preferentially in cells that transport dopamine ${ }^{13}$. Following recognition of MPTP's toxicity and its mechanism of action, several laboratories reported a selective defect in complex I of the electron transport chain in $\mathrm{PD}^{14-22}$. This defect seems to be systemic, affecting not only the brain, but also peripheral tissues such as platelets.

An accurate in vivo experimental model of PD should reproduce the progressive, selective nigrostriatal dopaminergic degeneration and Lewy body formation seen in PD, test the relevance of the systemic complex I defect, and explain the potential involvement of pesticide exposure in development of parkinsonism. Unfortunately, no current animal model incorporates all of these features. The MPTP model causes selective nigrostriatal degeneration by inhibiting complex I, but, unlike PD, MPTP does not cause a systemic complex I defect. Instead, the inhibition is highly selective for dopaminergic neurons. Moreover, MPTP does not typically produce cytoplasmic inclusions that closely resemble Lewy bodies $^{23}$. Transgenic mice expressing the pathogenic human $\alpha$-synuclein mutation develop modest dopaminergic pathology (although the specificity of this degeneration is not detailed), and many neurons contain small cytoplasmic inclusions that are granular rather than fibrillar ${ }^{24}$.

To develop a more accurate in vivo model of PD, we exposed rats chronically, continuously and systemically to the common pesticide, rotenone. A naturally occurring compound derived from the roots of certain plant species, rotenone is commonly used as an insecticide in vegetable gardens, and is also used to kill or sample fish populations in lakes and reservoirs. It is widely believed to be a safe, natural alternative to synthetic pesticides. Rotenone is also a well characterized, high-affinity, specific inhibitor of complex I, one of the five enzyme complexes of the inner mitochondrial membrane involved in oxidative phosphorylation. Because it is extremely hydrophobic, rotenone crosses biological membranes easily, and it does not depend on the dopamine transporter for access to the cytoplasm. Therefore, rotenone-unlike MPTP - is well-suited to produce a systemic inhibition of complex I.

\section{RESULTS}

Sprague-Dawley and Lewis rats were infused continuously with rotenone by a jugular vein cannula attached to a subcutaneous osmotic minipump. Lewis rats showed less variability and more consistent lesions than Sprague-Dawley rats ${ }^{25}$ and were used exclusively after completion of pilot studies. Initially, rotenone was infused at doses ranging from 1 to $12 \mathrm{mg} / \mathrm{kg}$ per day for various lengths of time. High doses of rotenone for short periods of time produced systemic (cardiovascular) toxicity and non-specific brain lesions, as reported by others ${ }^{26}$ (M.F. Beal and J. Shulz, personal 

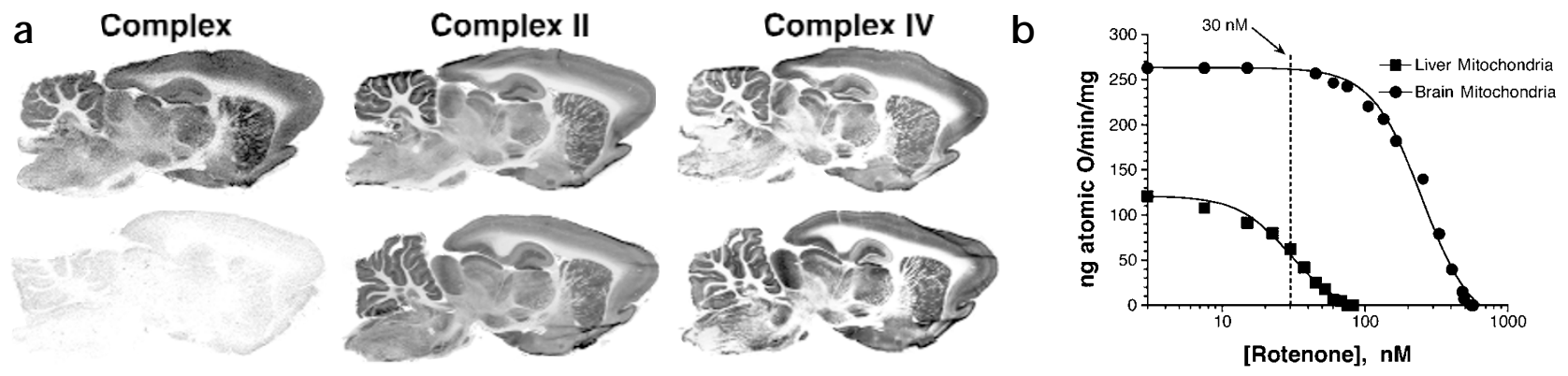

Fig. 1. Rotenone infusion selectively and uniformly affected complex I throughout the brain. (a) Histochemical analysis of mitochondrial complexes. Top, adjacent sections from a vehicle-infused animal. Bottom, adjacent sections from a rotenone-infused animal (2.0 mg/kg per day for 2 days). $\left[{ }^{3} \mathrm{H}\right.$ ] D ihydrorotenone binding to complex $\mathrm{I}^{28}$ and succinate dehydrogenase (complex II) and cytochrome oxidase (complex IV) histochemistry were done ${ }^{27}$. Marked reduction of complex I binding (73\%) indicated that these binding sites were occupied by infused rotenone. Based on this degree of binding inhibition, the rotenone concentration in brain was estimated to be $20-30 \mathrm{nM}^{27}$. Complexes II and IV were unchanged by rotenone infusion. (b) Titration of glutamate-supported respiration with rotenone in mitochondria from liver and brain. 0 ximetry 41,42 studies show that the estimated brain concentration of rotenone (30 nM maximum), known to partially inhibit complex I activity, partially inhibits respiration in liver mitochondria. However, it does not affect respiration in brain mitochondria.

communication). Downward titration of rotenone dosing resulted in less systemic illness and highly specific dopaminergic degeneration. The optimal dose for inducing the pathology of PD was determined to be $2-3 \mathrm{mg} / \mathrm{kg}$ per day in Lewis rats, and animals were euthanized after 7 days to more than 5 weeks of continuous treatment. In the study presented here, 25 rats were infused with rotenone in this dose range, and 12 demonstrated clear nigrostriatal dopaminergic lesions; no vehicle-treated rats had lesions.

Rotenone infusion affected complex I selectively. Enzymatic activities of succinate dehydrogenase (complex II) and cytochrome oxidase (complex IV), analyzed histochemically ${ }^{27}$, were not affected by rotenone infusion. In animals treated sys- temically with rotenone, $\left[{ }^{3} \mathrm{H}\right]$ dihydrorotenone binding to complex I in brain ${ }^{28}$ was reduced by about $75 \%$ (Fig. 1a). Systemically administered rotenone acted on complex I uniformly throughout the brain, consistent with its ability to cross biological membranes easily. Based on the affinities of rotenone and dihydrorotenone for complex I, a 75\% inhibition of specific binding translates to a free rotenone concentration of $20-30 \mathrm{nM}$ in the brain ${ }^{28}$. This concentration is known to partially inhibit complex I activity ${ }^{29}$. However, oximetry analysis indicated that in brain mitochondria-but not liver mitochondria - this level of complex I inhibition was insufficient to inhibit glutamate-supported respiration (Fig. 1b).

Fig. 2. Systemic rotenone infusion resulted in nigrostriatal dopaminergic degeneration. Coronal brain sections from control (a, e) and rotenoneinfused rats ( $2.5 \mathrm{mg} / \mathrm{kg}$ per day; (b-d, $\mathbf{f}-\mathbf{h})$ were immunostained for TH. Duration of the rotenone infusion was 7 days (b, f), 36 days (c, $\mathbf{g})$ or 33 days (d) h). (a-d) TH immunocytochemistry in striatum. (a) Vehicle-infused rat. (b-d) Rotenone-infused rats. Progressive striatal dopaminergic denervation (arrows), ranging from partial $(\mathbf{b}, \mathbf{c})$ to almost complete (d) was observed. D espite the almost total loss of dopaminergic terminals in the motor striatum, dopaminergic fibers were spared in the medial striatum, nucleus accumbens and olfactory tubercle (b-d). Scale bar, 2 mm. ctx, cortex; st, striatum; na, nucleus accumbens; ot, olfactory tubercle. (e-h) TH immunocytochemistry in substantia nigra. (e) Vehicle-infused rat. (f, g) Rotenoneinfused rats with partial striatal denervation maintained relatively normal TH staining in substantia nigra. (h) W ith more substantial striatal denervation, there was a loss of TH-immunoreactive neurons and processes. Neurons in the lateral portion (arrows) and ventral tier (arrowheads) of substantia nigra pars compacta were most vulnerable. Scale bar, $1 \mathrm{~mm}$. SN c, substantia nigra pars compacta; VTA, ventral tegmental area.

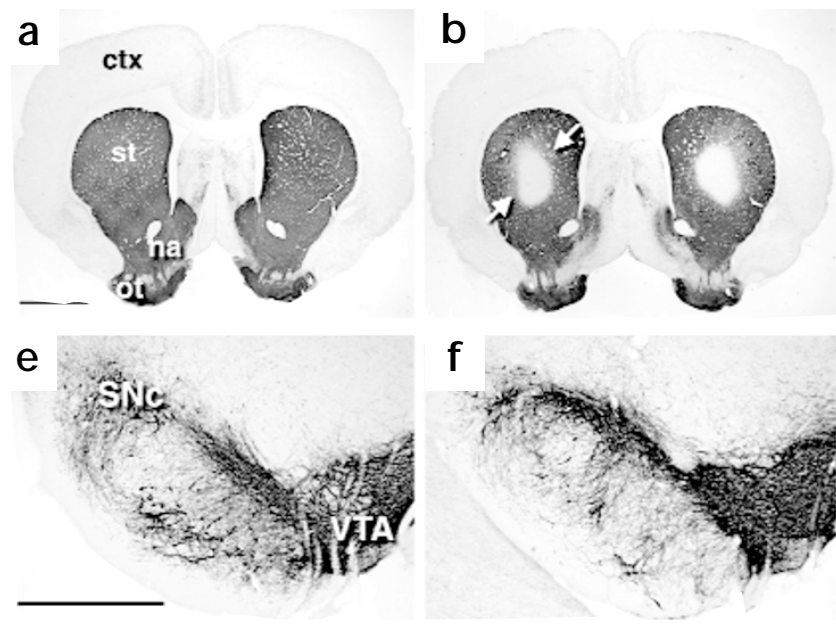

C<smiles>[CH3-]</smiles>

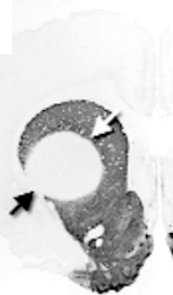

g

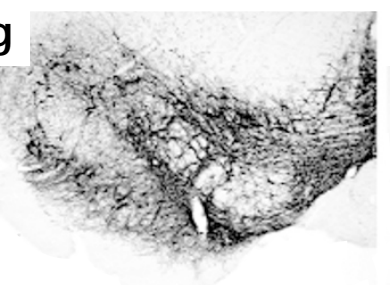


Fig. 3. D egeneration of nerve terminals and cell bodies of nigrostriatal dopaminergic neurons in perfusion-fixed brain sections. Sections were taken from rats that received $2.5-2.75 \mathrm{mg} / \mathrm{kg}$ per day rotenone for $1-5$ weeks. (a, b) Silver staining in the striatum. (a) In a rat with a partial lesion (2.5 mg/kg per day for 7 days), silver deposits (gray material denoted by red arrows) were restricted to focal regions devoid of TH immunoreactivity (brown reaction product). Silver grains and deposits indicate the presence of degenerating nerve terminals. Unaffected striatal regions and cortex lacked silver grains. ctx, cortex. Scale bar, $1 \mathrm{~mm}$. (b) In a rat with an almost complete lesion $(2.5 \mathrm{mg} / \mathrm{kg}$ per day for 33 days), there was widespread silver staining throughout the striatum (red arrows). Scale bar, $200 \mu \mathrm{m}$. (c-f) Silver staining in substantia nigra. (c) In vehicle-infused rats, silver deposits were never seen in cell bodies or processes. (d, e) $\mathrm{N}$ igral neurons containing silver grains were present, along with normal cells, in rotenone-treated animals with partial denervation of striatum. Arrowheads indicate presence of silver grains in dendrites. (f) Silver grains were present throughout cell bodies and dendrites of degenerating nigral neurons in rats with near complete denervation of striatum. Scale bar, $10 \mu \mathrm{m}$. ( $\mathbf{g}$ h) Fluoro-Jade B histochemistry in substantia nigra. (g) In vehicle-infused rats, no Fluoro-Jade B positive neurons were detected. (h) In rotenone-treated rats, numerous Fluoro-Jade B positive neurons (white arrows) were seen in substantia nigra. Scale bar, $30 \mu \mathrm{m}$.

\section{Selective nigrostriatal dopaminergic degeneration}

Systemic partial inhibition of complex I resulted in progressive nigrostriatal dopaminergic degeneration. Immunocytochemistry of phenotypic markers for dopaminergic neurons (tyrosine hydroxylase, TH; dopamine transporter, DAT; vesicular monoamine transporter type 2, VMAT2) all gave identical results, indicating dopaminergic degeneration. Depending on the dose and duration of rotenone exposure, animals demonstrated varying degrees of striatal dopaminergic denervation (Fig. 2b-d). Lesions typically began focally in the central or dorsolateral portion of the anterior striatum, as evidenced by a complete loss of phenotypic markers, and spread to involve most of the motor striatum. Even when striatal TH depletion was almost complete, there was relative sparing of dopaminergic fibers in medial aspects of striatum, nucleus accumbens and olfactory tubercle (Fig. 2d), areas that are relatively spared in idiopathic $\mathrm{PD}^{30}$.

There was also evidence of dopaminergic lesions in the cell bodies of the substantia nigra pars compacta. Animals with partial reductions in $\mathrm{TH}$ staining in striatum had dopaminergic neurons in substantia nigra that looked relatively normal (Fig. $2 \mathrm{f}$ and g); however, in animals with near complete striatal denervation, there were obvious reductions in $\mathrm{TH}$-stained cells in substantia nigra (Fig. $2 \mathrm{~h}$ ). These results suggest that striatal nerve terminals were affected earlier and more severely by rotenone than nigral cell bodies. Similarly, in brains of dying patients with PD, there is a more profound loss of dopamine in striatum compared to substantia nigra $^{31}$. Neurons in the lateral portion and ventral tier of the substantia nigra pars compacta were most vulnerable to systemic rotenone infusion (Fig. $2 \mathrm{~h}$ ). This pattern of vulnerability matches the pattern in idiopathic PD. Despite the loss of TH staining in

Fig. 4. Striatal neurons postsynaptic to the degenerating dopaminergic fibers were unaffected by systemic rotenone infusion. (a-d) Sections of striatum from a control animal $(\mathbf{a}, \mathbf{c})$ and an animal that received $2.5 \mathrm{mg} / \mathrm{kg}$ per day rotenone for 33 days $(\mathbf{b}, \mathbf{d})$ were double-labeled for $\mathrm{TH}(\mathbf{a}, \mathbf{b})$ and GAD $(\mathbf{c}, \mathbf{d})$. In regions of striatum that completely lacked TH immunoreactivity (b), GAD immunofluorescence was intact (d). Scale bar, $50 \mu \mathrm{m}$. (e, f) AChE activity was unaffected in animals with dopaminergic denervation. Scale bar, $200 \mu \mathrm{m}$.
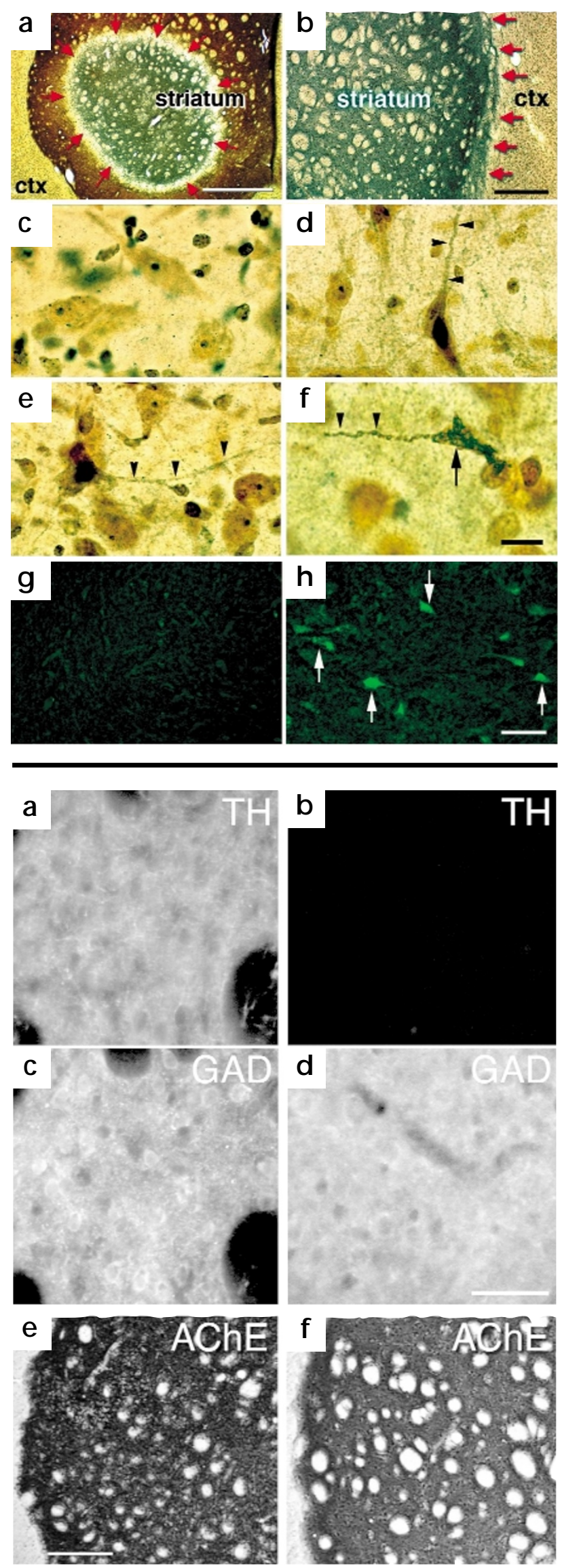


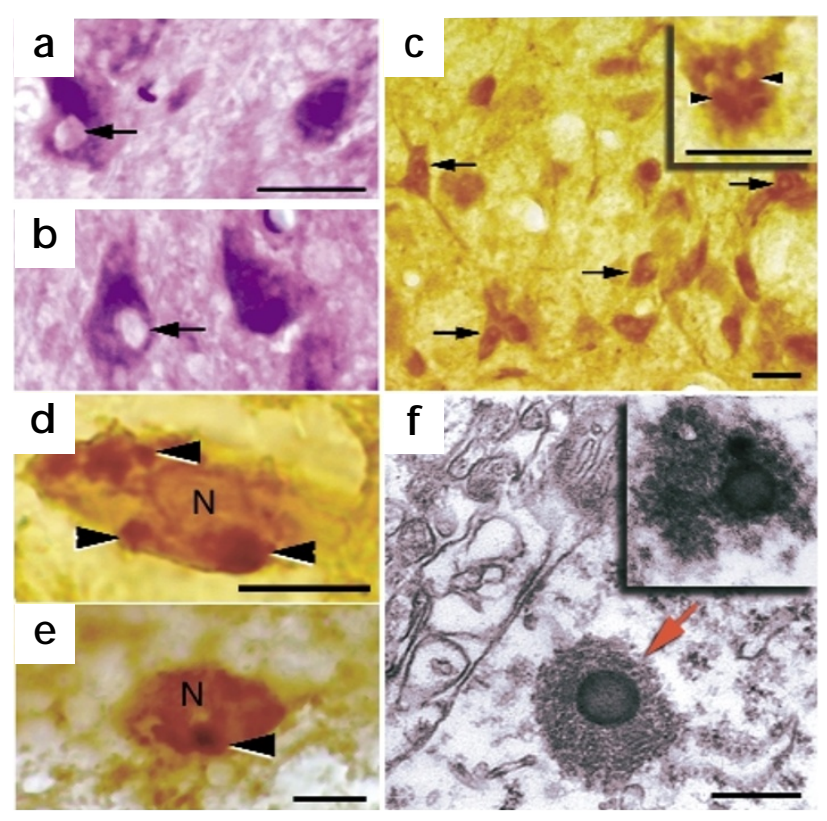

Fig. 5. Cytoplasmic inclusions in nigral neurons of rotenone-infused rats. (a, b) Pale cytoplasmic inclusions (arrows) were seen in nigral neurons of rotenone-infused rats by standard hematoxylin and eosin staining. Scale bar, $25 \mu \mathrm{m}$. (c) Cytoplasmic inclusions in nigral neurons contained ubiquitin immunoreactivity. Arrows indicate cells containing ubiquitin-positive aggregates. Inset, a nigral cell at higher magnification illustrating ubiquitinpositive inclusions (arrowheads). Scale bars, $25 \mu \mathrm{m}$. (d, e) N eurons with $\alpha$-synuclein-positive inclusions (arrowheads) Scale bars, $10 \mu \mathrm{m}$. N , nucleus. (f) UItrastructural analysis of inclusions showed a dense core with peripheral fibrillar elements (arrow). Inset, an $\alpha$-synuclein-positive inclusion as demonstrated by immuno-electron microscopy. Scale bar, $1 \mu \mathrm{m}$.

substantia nigra, dopaminergic neurons of the ventral tegmental area (VTA) were relatively spared, as they are in PD. Moreover, just as in idiopathic PD, noradrenergic neurons of the locus ceruleus were mildly to moderately affected (data not shown).

To confirm that the loss of staining of dopaminergic neurons and nerve terminals was due to degeneration rather than reduced expression of phenotypic markers by surviving cells, silver staining was done alone and in combination with TH immunostaining. In the striatum, regions of degeneration densely stained with silver corresponded to areas where there was loss of TH staining (Fig. 3a and b). In the substantia nigra, there was also clear degeneration. Although animals treated with vehicle never showed nigral degeneration (Fig. 3c), animals with partial loss of striatal dopamine terminals had many nigral neurons with silver deposits in their cell bodies or dendrites, interspersed with neurons that looked normal (Fig. 3d and e). Thus, although nigral TH staining was relatively normal in animals with partial denervation of striatum, silver staining revealed degenerative changes. Animals with severe dopaminergic denervation of striatum had extensive silver deposition in substantia nigra corresponding to the loss of phenotypic markers in this region (Fig. 3f). Silver staining also confirmed the progressive, retrograde nature of dopaminergic degeneration in the rotenone model. In an animal that survived for two weeks beyond the end of rotenone infusion, there was no longer silver staining in the region of striatum depleted of dopaminergic markers. Presumably, this was due to previous phagocytosis of degenerating neuronal elements. However, in this animal, there was massive silver deposition in substantia nigra (data not shown). Silver staining also showed that there was no consistent neuronal degeneration elsewhere in the brains of rotenone-treated animals. For example, there was no silver staining in the cortex in Fig. 3b. Although we never saw silver staining in control animals, there was the possibility that silver deposition could reflect protein aggregation in the absence of frank degeneration. Therefore, the neuronal degeneration was confirmed in rotenone-infused animals with Fluoro-Jade B histochemistry. Fluoro-Jade B is a fluorescein derivative that selectively stains neurons undergoing degeneration ${ }^{32}$. Vehicle-infused animals had no Fluoro-Jade B-positive neurons in substantia nigra (Fig. 3g); however, there were numerous Fluoro-Jade Bpositive neurons in substantia nigra of rotenone-infused animals (Fig. 3h). Together, silver staining and Fluoro-Jade B histochemistry conclusively demonstrated selective degeneration of nigrostriatal dopamine neurons in rotenone-infused animals.

Despite the profound loss of presynaptic dopaminergic nerve terminals in striatum, postsynaptic neuronal elements in striatum remained intact. Nissl staining, though relatively insensitive in striatum, did not show obvious lesions of striatal neurons (data not shown). More than $90 \%$ of striatal neurons use $\gamma$-aminobutyric acid as their transmitter, and they can be identified with immunocytochemistry for glutamic acid decarboxylase (GAD). In striatal regions lacking dopaminergic terminals, there was no loss of GAD immunoreactivity (Fig. 4a-d). Thus, the predominant cell type in striatum seemed to be intact in rotenone-treated animals. This is in direct contrast to what occurs when complex II-rather than complex I-is inhibited systemically. Animals treated systemically with the complex II inhibitor, 3-nitropropionic acid, display selective degeneration of striatal neurons with relative sparing of the nigrostriatal dopamine system ${ }^{33}$. An additional smaller population of striatal neurons is cholinergic, and can be assessed with acetylcholinesterase (AChE) staining ${ }^{34}$. Striatal AChE staining was similar in vehicle- and rotenone-treated animals (Fig. 4e and $\mathrm{f}$ ). These results further support the nigrostriatal dopaminergic selectivity of rotenone-induced neurodegeneration.

\section{Cytoplasmic inclusions reminiscent of Lewy bodies}

Rats with dopaminergic degeneration had cytoplasmic inclusions in nigral neurons. These inclusions shared some features of the Lewy bodies associated with $\mathrm{PD}^{1}$. With hematoxylin and eosin staining, pale eosinophilic inclusions were seen that resembled the 'pale body' precursors to Lewy bodies (Fig. $5 \mathbf{a}$ and $\mathbf{b}$ ). With light microscopy, we saw some nigral neurons that also showed aggregates (usually $1-4 \mu \mathrm{m}$ in diameter) containing ubiquitin (Fig. 5c) and $\alpha$-synuclein (Fig. 5d and e). Electron microscopy showed electron-dense cytoplasmic inclusions (1-2 $\mu \mathrm{m}$ in diameter) in the cytoplasm of nigral neurons (Fig. 5f); such inclusions were frequently clustered in the cytoplasm. Ultrastructural features included a homogeneous dense core surrounded by fibrillar elements similar to those seen in Lewy bodies. Some of the inclusions had a more granular appearance, like those described in $\alpha$-synuclein transgenic mice ${ }^{24}$. By immuno-electron microscopy, we saw that these inclusions contained $\alpha$-synuclein immunoreactivity (Fig. 5f, inset). We did not find neuronal inclusions in rotenone-treated animals that did not have dopaminergic lesions as assessed by $\mathrm{TH}$ staining.

\section{Parkinsonian behavior}

Initial characterization of this model focused on delineating the biochemical, molecular and anatomical pathology of rotenoneinduced degeneration. However, rotenone-treated animals developed motor and postural deficits characteristic of PD, the severity 
of which depended on the extent of lesions. All animals with a dopaminergic lesion became hypokinetic and had unsteady movement and hunched posture, even after termination of the rotenone infusion. Seven animals developed severe rigidity, and three animals had shaking of one or more paws that was reminiscent of rest tremor. Preliminary experiments in two animals indicate that the motor deficits were ameliorated by the dopamine agonist, apomorphine (data not shown).

\section{Discussion}

Taken together, our results indicate that a systemic partial defect in complex I is enough to reproduce the behavioral, anatomical, neurochemical and neuropathological features of PD. The finding that rotenone affected complex I uniformly throughout the brain (Fig. 1a), coupled with the resultant highly selective neurodegeneration of the nigrostriatal dopaminergic system, suggested that this population of neurons had an intrinsic sensitivity to complex I defects. Because the brain rotenone concentration (20-30 nM) was enough to partially inhibit complex I, but too low to significantly impair respiration of brain mitochondria, it seemed that a bioenergetic defect with ATP depletion could not explain the neurodegneration. Instead, oxidative damage might have been involved. Inhibition of complex I by rotenone stimulates production of reactive oxygen species ${ }^{35,36}$. Moreover, we have found that culture of neural cells with $5 \mathrm{nM}$ rotenone induces, over a period of weeks, progressive oxidative damage to proteins and DNA, and sensitizes cells to subsequent oxidative stressors; eventually, it also begins to induce release of cytochrome c from mitochondria to the cytoplasm (Sherer et al., Soc. Neurosci. Abstr. 26, 280.21, 2000). This mechanism could also explain the cytoplasmic inclusions found in nigral neurons of rotenonetreated rats, because both oxidative damage and cytochrome $c$ enhance $\alpha$-synuclein aggregation ${ }^{37,38}$.

Rotenone seems to have little toxicity when administered orally (T. B. S. and J. T. G., unpublished results); however, our results highlight the possibility that environmental toxins, including pesticides that inhibit mitochondrial function, may contribute to the pathogenesis of PD. Many other naturally occurring compounds and synthetic pesticides are potent inhibitors of complex I ${ }^{39}$. Individuals are likely to be variably exposed to numerous natural or synthetic complex I inhibitors through diet, drinking water or other environmental factors. Such exposures, combined with genetic differences in complex I function ${ }^{14-22}$, or inter-individual differences in the ability to metabolize xenobiotics ${ }^{7}$, may underlie most cases of typical idiopathic PD.

\section{Methods}

Animal surgery. We used male Sprague Dawley and Lewis rats (300-350 g; approximately 2 months old) for this study. Alzet osmotic mini pumps (models $2 \mathrm{ML} 4$ or $2 \mathrm{ML} 1$ ) were filled with rotenone, dissolved in equal volumes of dimethylsulfoxide (DMSO) and polyethylene glycol (PEG). Pumps were attached to Tygon microbore (0.58 mm i.d.) tubing (Fisher Scientific, Pittsburgh, Pennsylvania) and placed in sterile $0.9 \%$ saline at $37^{\circ} \mathrm{C}$ for at least $4 \mathrm{~h}$. Ketamine $(75 \mathrm{mg} / \mathrm{kg})$ and Rompum $(10 \mathrm{mg} / \mathrm{kg})$ were injected intramuscularly for anesthetic. Alzet osmotic mini pumps were implanted under the skin on the back of each animal, and the right jugular vein was cannulated. Pumps were exchanged after 28 days. Control rats received DMSO:PEG (1:1) only. Following surgery, rats were monitored for behavior, weight loss and overall health. In the instances when rotenone-treated rats began to lose weight, diet was supplemented with oral administration of Nutrical (Evsco Pharmaceuticals, Buena, New Jersey). Subcutaneous lactated Ringer's injection USP solution (Baxter Healthcare Corporation, Deerfield, Illinois) was given when rats showed signs of dehydration.
Mitochondrial respiration. Oxygen consumption by rat brain and liver mitochondria was measured polargraphically as described ${ }^{40}$ using an Instech minichamber (Instech Laboratories, Plymouth Meeting, Pennsylvania) equipped with a magnetic stirrer and oxygen electrode (Yellow Spring Instrument Company, Yellow Springs, Ohio) connected to a chart recorder. For maximum State 3 respiration activity, the following medium was used: $125 \mathrm{mM} \mathrm{KCl}, 10 \mathrm{mM}$ MOPS, $2 \mathrm{mM} \mathrm{MgCl}_{2}, 2 \mathrm{mM}$ $\mathrm{KH}_{2} \mathrm{PO}_{4}, 1 \mathrm{mM}$ EGTA, $0.7 \mathrm{mM} \mathrm{CaCl}, 20 \mathrm{mM}$ glucose, 8 units of hexokinase, and $20 \mathrm{mM}$ glutamate plus $2 \mathrm{mM}$ malate. Brain mitochondria were isolated by the method of Sims ${ }^{41}$ without BSA, because BSA binds rotenone nonspecifically.

Immunocytochemistry. We used paraformaldehyde-fixed brains. For single immunolabeling studies, $40-\mu \mathrm{m}$ sections were incubated in primary antibody for $24 \mathrm{~h}$, followed by $1 \mathrm{~h}$ incubation with biotinylated secondary antibody. The avidin-biotin complex method was used to detect the antigen signal (ABC elite kit, Vector laboratories, Burlingame, California) and 3,3'-diaminobenzidine tetrachloride (DAB) was used to visualize the final product. The primary antibodies used were monoclonal mouse antibody against TH (1:2000; Chemicon, Temecula, California), polyclonal rabbit antibody against ubiquitin (1:1000; Dako Corporation, Carpinteria, California) and polyclonal rabbit antibody against $\alpha$-synuclein (1:400). Secondary antibodies used were biotinylated goat anti-mouse immunoglobulin $\mathrm{G}(\operatorname{IgG})$ or biotinylated goat anti-rabbit $\operatorname{IgG}(1: 200$; Jackson Immunoresearch Labs, West Grove, Pennsylvania). Double labeling for TH and GAD was performed by immunofluorescence. Sections were simultaneously incubated with antibodies against TH (1:2000; Chemicon) and GAD (1:2000; polyclonal rabbit antibody; Chemicon) for $24 \mathrm{~h}$. Texas red-conjugated goat anti-mouse IgG was used to detect TH-positive cells and fluorescein isothiocyanate-conjugated goat anti-rabbit IgG was used to detect GAD- expressing cells (1:200; Jackson Immunoresearch Labs). For controls, one or both primary antibodies were omitted. We examined immunostained sections using bright-field microscopy or conventional fluorescence microscopy. We captured images on a Leitz microscope (Leica, Wetzlar, Germany) linked to an image analysis system (Imaging Research, St. Catharines, Ontario, Canada) with selective filter sets to visualize FITC and Texas red separately, as well as simultaneously. For final output, images were processed using Adobe Photoshop 5.0 software.

Detection of neuronal degeneration. Both silver staining and FluoroJade B histochemistry were used to detect degenerating neurons. The FD Neurosilver kit (FD NeuroTechnologies, Ellicott City, Maryland) stained degenerating neuronal elements in paraformaldehyde-fixed brain tissue from control and rotenone-infused rats. Silver staining was done according to the manufacturer's protocol. Following silver staining, sections were mounted on Superfrost plus slides (Fisher Scientific, Pittsburgh, Pennsylvania), air-dried, cleared with xylene, and coverslipped with Permount. Sections were examined using bright-field microscopy. TH-immunostained sections were also processed for silver staining to demonstrate selective neurodegeneration. Fluoro-Jade B histochemistry was done on paraformaldehyde-fixed brain sections from control and rotenone-infused rats according to the manufacturer's protocol (HistoChem, Jefferson, Arkansas).

Electron microscopy. Paraformaldehyde-fixed brain sections from four rats were processed for standard electron microscopy. The tissue was post-fixed in osmium tetroxide and embedded in epon. Ultra-thin sections were stained with uranyl acetate and lead citrate and examined with a Hitachi (Mountain View, California) electron microscope. For immuno-electron microscopy, sections stained for $\alpha$-synuclein using $\mathrm{DAB}$ as chromophore were fixed with $0.5 \%$ glutaraldehyde and processed for routine electron microscopy as described.

\section{ACKNOWLEDGEMENTS}

We thank P. Piccardo and B. Ghetti for donation of the $\alpha$-synuclein antibody, which was generated with support from the Indiana Alzheimer's Disease Center (P30AG10133). We also thank M. DeLong and A. Levey for reading this manuscript. This work was supported by NIH grants to JTG (NS38399, NS33779, and AG14648). 


\section{articles}

\section{ReCEIVED 7 SEPTEMBER; ACCEPTED 25 OCTOBER 2000}

1. Baba, M. et al. Aggregation of alpha-synuclein in Lewy bodies of sporadic Parkinson's disease and dementia with Lewy bodies. Am. J. Pathol. 152, 879-884 (1998).

2. Kruger, R. et al. Ala30Pro mutation in the gene encoding alpha-synuclein in Parkinson's disease. Nat. Genet. 18, 106-108 (1998).

3. Polymeropoulos, M. H. et al. Mutation in the alpha-synuclein gene identified in families with Parkinson's disease. Science 276, 2045-2047 (1997).

4. Kitada, T. et al. Mutations in the parkin gene cause autosomal recessive juvenile parkinsonism. Nature 392, 605-608 (1998).

5. Tanner, C. M. et al. Parkinson disease in twins: an etiologic study. JAMA 281, 341-346 (1999).

6. Dexter, D. T. et al. Basal lipid peroxidation in substantia nigra is increased in Parkinson's disease. J. Neurochem. 52, 381-389 (1989).

7. Menegon, A., Board, P. G., Blackburn, A. C., Mellick, G. D. \& Le Couteur, D. G. Parkinson's disease, pesticides, and glutathione transferase polymorphisms. Lancet 352, 1344-1346 (1998).

8. Butterfield, P. G., Valanis, B. G., Spencer, P. S., Lindeman, C. A. \& Nutt, J. G. Environmental antecedents of young-onset Parkinson's disease. Neurology 43, 1150-1158 (1993).

9. Gorell, J. M., Johnson, C. C., Rybicki, B. A., Peterson, E. L. \& Richardson, R. J. The risk of Parkinson's disease with exposure to pesticides, farming, well water, and rural living. Neurology 50, 1346-1350 (1998).

10. Langston, J. W., Ballard, P. A., Tetrud, J. W. \& Irwin, I. Chronic parkinsonism in humans due to a product of meridine-analog synthesis. Science 219, 979-980 (1983).

11. Nicklas, W. J. \& Heikkila, R. E. Inhibition of NADH-linked oxidation in brain mitochondria by 1-methyl-4-phenylpyridine, a metabolite of the neurotoxin, 1-methyl-4-phenyl-1,2,3,6-tetrahydropyridine. Life Sci. 36, 2503-2508 (1985).

12. Tipton, K. F. \& Singer, T. P. Advances in our understanding of the mechanisms of the neurotoxicity of MPTP and related compounds. J. Neurochem. 61, 1191-1206 (1993).

13. Tolwani, R. J., Jakowec, M. W., Petzinger, G. M., Green, S. \& Waggie, K. Experimental models of Parkinson's disease: insights from many models. Lab. Anim. Sci. 49, 363-371 (1999).

14. Parker, W. D., Boyson, S. J. \& Parks, J. K. Abnormalities of the electron transport chain in idiopathic Parkinson's disease. Ann. Neurol. 26, 719-723 (1989).

15. Bindhoff, L. A., Birch-Machin, M., Cartlidge, N. E., Parker, W. D. Jr. \& Turnbull, D. M. Mitochondrial function in Parkinson's disease. Lancet 2, 49 (1989).

16. Schapira, A.H. et al. Mitochondrial complex I deficiency in Parkinson's disease. J. Neurochem. 54, 823-827 (1990).

17. Shoffner, J. M., Watts, R. L., Juncos, J. L., Torroni, A. \& Wallace, D. C. Mitochondrial oxidative-phosphorylation defects in Parkinson's disease. Ann. Neurol. 30, 332-339 (1991).

18. Mann, V. M. et al. Brain, skeletal-muscle and platelet homogenate mitochondrial function in Parkinson's disease. Brain 115, 333-342 (1992).

19. Cardellach, F. et al. Mitochondrial respiratory-chain activity in skeletal muscle from patients with Parkinsons disease. Neurology 43, 2258-2262 (1993).

20. Blin, O. et al. Mitochondrial respiratory failure in skeletal muscle from patients with Parkinson's disease and multiple system atrophy. J. Neurol. Sci. 125, 95-101 (1994).

21. Swerdlow, R. H. et al. Matrilineal inheritance of complex I dysfunction in a multigenerational Parkinson's disease family. Ann. Neurol. 44, 873-881 (1998).

22. Mizuno, Y. et al. Mitochondrial dysfunction in Parkinson's disease. Ann. Neurol. 44 (3 Suppl. 1), S99-109 (1998).
23. Forno, L. S., DeLanney, L. E., Irwin, I. \& Langston, J. W. Electron microscopy of Lewy bodies in the amygdala-parahippocampal region. Comparison with inclusions bodies in the MPTP-treated squirrel monkey. Adv. Neurol. 69, 217-228 (1996).

24. Masliah, E. et al. Dopaminergic loss and inclusion body formation in $\alpha$-synuclein mice: implications for neurodegenerative disorders. Science 287, 1265-1269 (2000).

25. Ouary, S. et al. Major strain differences in response to chronic systemic administration of the mitochondrial toxin 3-nitropropionic acid in rats: implications for neuroprotection studies. Neuroscience 97, 521-530 (2000).

26. Ferrante, R. J., Schluz, J. B., Kowall, N. W. \& Beal, M. F. Systemic administration of rotenone produces selective damage in the striatum and globus pallidus, but not the substantia nigra. Brain Res. 753, 157-162 (1997).

27. Porter, R. H. P., Greene, J. G., Higgins, D. S. Jr. \& Greenamyre, J. T. Polysynaptic regulation of glutamate receptors and mitochondrial enzyme activities in the basal ganglia of rats with unilateral dopamine depletion. J. Neurosci. 14, 7192-7199 (1994).

28. Higgins, D. S. Jr. \& Greenamyre, J. T. $\left[{ }^{3} \mathrm{H}\right]$ Dihydrorotenone binding to NADH: Ubiquinone reductase (complex I) of the electron transport chain: an autoradiographic study. J. Neurosci. 16, 3807-3816 (1996).

29. Davey, G. P. \& Clark, J. B. Threshold effects and control of oxidative phosphorylation in nonsynaptic rat brain mitochondria. J. Neurochem. 66, 1617-1624 (1996).

30. Miller, G. W. et al. Immunochemical analysis of dopamine transporter protein in Parkinson's disease. Ann. Neurol. 41, 530-539 (1997).

31. Hornykiewicz, O. Dopamine (3-hydroxytyramine) and brain function. Pharmacol. Rev. 18, 925-965 (1966).

32. Schmued, L. C., Albertson, C. \& Slikker, W. Jr. Fluoro-Jade: a novel fluorochrome for the sensitive and reliable histochemical localization of neuronal degeneration. Brain Res. 751, 37-46 (1997).

33. Beal, M. F. et al. Neurochemical and histologic characterization of striatal excitotoxic lesions produced by the mitochondrial toxin 3-nitropropionic acid. J. Neurosci. 13, 4181-4192 (1993).

34. Hedreen, J. C., Bacon, S. J. \& Price, D. L. A modified histochemical technique to visualize acetylcholinesterase-containing axons. J. Histochem. Cytochem. 33, 134-140 (1985).

35. Hensley, K. et al. Interaction of alpha-phenyl-N-tert-butyl nitrone and alternative electron acceptors with complex I indicates a substrate reduction site upstream from the rotenone binding site. J. Neurochem. 71, 2549-2557 (1998).

36. Seaton, T. A., Cooper, J. M. \& Schapira, A. H. Free radical scavengers protect dopaminergic cell lines from apoptosis induced by complex I inhibitors. Brain Res. 777, 110-118 (1997).

37. Souza, J. M., Giasson, B. I., Chen, Q., Lee, V. M.-Y \& Ischiropoulos, H. Dityrosine cross-linking promotes formation of stable $\alpha$-synuclein polymers. J. Biol. Chem. 275, 18344-18349 (2000).

38. Hashimoto, M., Takeda, A., Hsu, L. J., Takenouchi, T. \& Masliah, E. Role of cytochrome $\mathrm{c}$ as a stimulator of $\alpha$-synuclein aggregation in Lewy body disease. J. Biol. Chem. 274, 28849-28852 (2000).

39. Degli Esposti, M. Inhibitors of NADH-ubiquinone reductase: an overview. Biochim. Biophys. Acta 1364, 222-235 (1998).

40. Panov, A. V. \& Scaduto, R. C. Jr. Substrate specific effects of calcium on metabolism of rat heart mitochondria. Am. J. Physiol. 270, H1398-H1406 (1996).

41. Sims, N. R. Rapid isolation of metabolically active mitochondria from rat brain and subregions using Percoll density gradient centrifugation. J. Neurochem. 55, 698-707 (1990). 